\title{
Pulsed-Phase Thermography for Thin-Film Photovoltaic Inspection
}

\author{
by H. Hoppe*, R. Meitzner*, H. Kruschke**, J.B. Slowik* and U.S. Schubert*
}

\begin{abstract}
* Center for Energy and Environmental Chemistry Jena (CEEC Jena) as well as Laboratory of Organic and Macromolecular Chemistry (IOMC), Friedrich Schiller University Jena, Philosophenweg 7a, D-07743 Jena, GERMANY, Harald.Hoppe@uni-jena.de

** InfraTec GmbH Infrarotsensorik und Messtechnik, Gostritzer Str. 61 - 63, D-01217 Dresden, GERMANY, H.Kruschke@infratec.de
\end{abstract}

\begin{abstract}
Traditionally, lock-in thermography is used for thermal investigation of photovoltaic devices. While this method provides excellent signal-to-noise ratios, it requires measurement times on the order of minutes. Such a method is therefore not suited for inline inspection of a photovoltaic production. Considerably lower measurement integration times are achieved for pulsed-phase thermography. We decided to test this method and apply it for inline inspection. First results were obtained on resting samples, which where illuminated by a flash light.
\end{abstract}

\section{Introduction}

We present initial results of the development of an inline defect detection and inspection tool for thin-film photovoltaic production. The technology is based on Pulsed-Phase Thermography. While the classical lock-in thermography allows for highly sensitive detection of heat dissipation processes and thus for the detection of peculiarities or defects within thin-film solar cells, such measurement commonly takes place on the order of minutes. The measurement time is a result of the periodic excitation by light or by voltage bias, which brings about the necessity to integrate over several cycles of excitations. While this method is excellent for off-line inspection, it is unfortunately not suited for in-line inspection due to vastly higher processing rates than the time required for such measurement. We are therefore developing a detection mode, which does not need such long integration times. In fact, by application of this contactless method to thin-film photovoltaic modules we could already demonstrate that the total measurement process can be achieved within less than a second. By comparison with classical imaging methods, we seek rules for unambiguous defect detection within different parts of the device. It is pursued not only to apply this method on final products, but also during intermediate production steps, which may as well allow for the detection of defects such as film inhomogeneities and coating or printing defects in general.

\section{Initial results}

By the usage of different imaging techniques, i.e. photo- and electroluminescence imaging, lock-in thermography and light beam induced current alongside our method under development, we are able to detect defects like missing interlayers and hot spots on commercially relevant printed solar modules [1]. While among these classical methods, light beam induced current (LBIC) is certainly the slowest with recording times of several hours, with the new technique, some information was possible to be obtained in just 20 ms due to the high excitation power.

In Figure 1 a comparison between an illuminated lock-in thermography (ILIT) image (left) and an image taken with the new method (right) are shown. So far it was possible to detect several of the defects seen by ILIT upon measuring for only 20 ms with our new method. Figure 2 shows some detailed light beam induced current mappings for 2 defect prone areas of the same module, which is shown in Figure 1. Cell 2 from the bottom has a highly reduced current generation in comparison to its neighbouring cells. The bottom cell (right panel Figure 2) did however operate normally, except for the areas of the holes. 


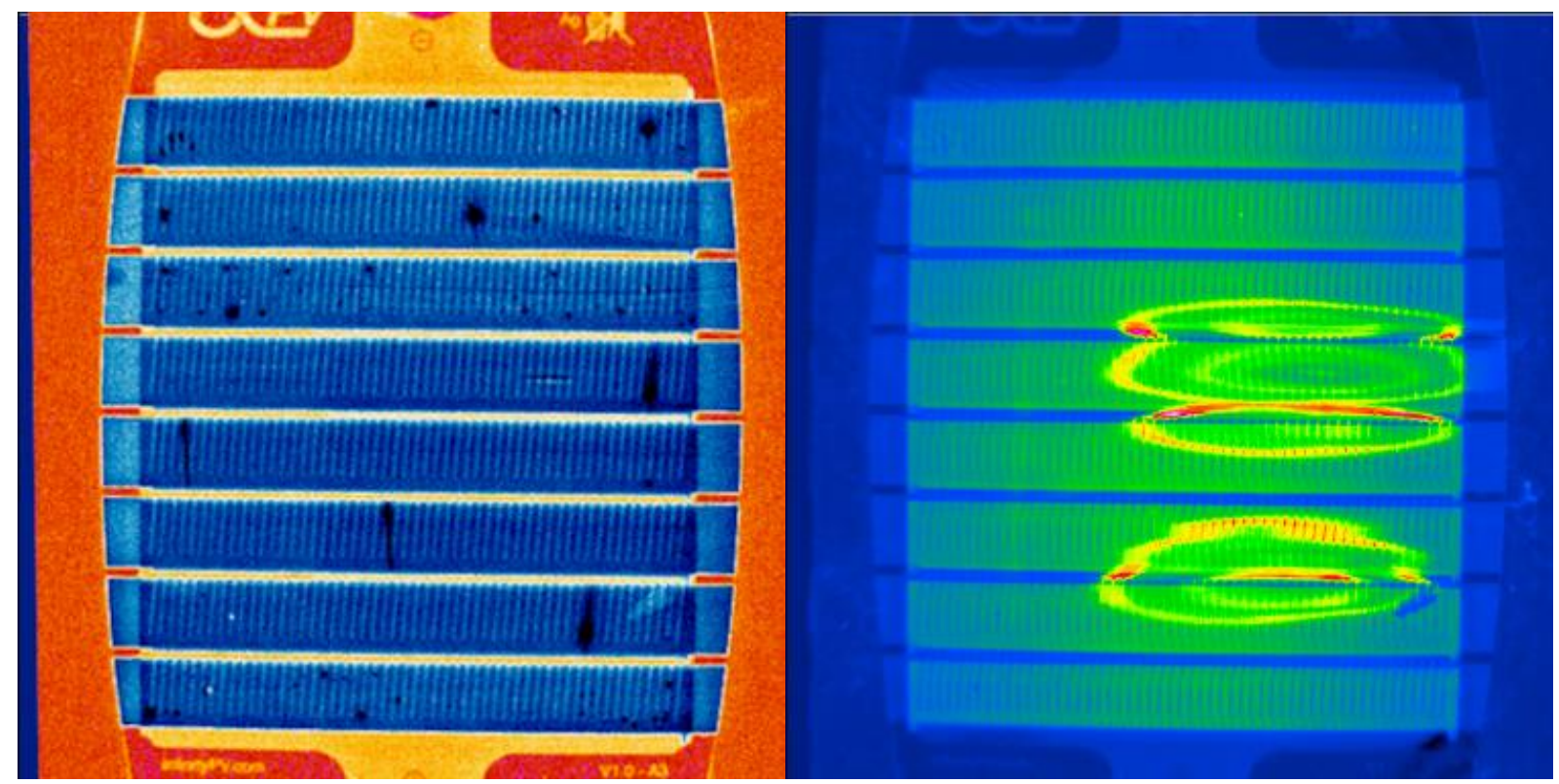

Fig. 1: Comparison of ILIT image (left) with the single image of the new method after $20 \mathrm{~ms}$ (right). Clearly seen on the right can be the missing transport layer (red circle), hot spots (yellow circles) and missing active layer (orange spots).
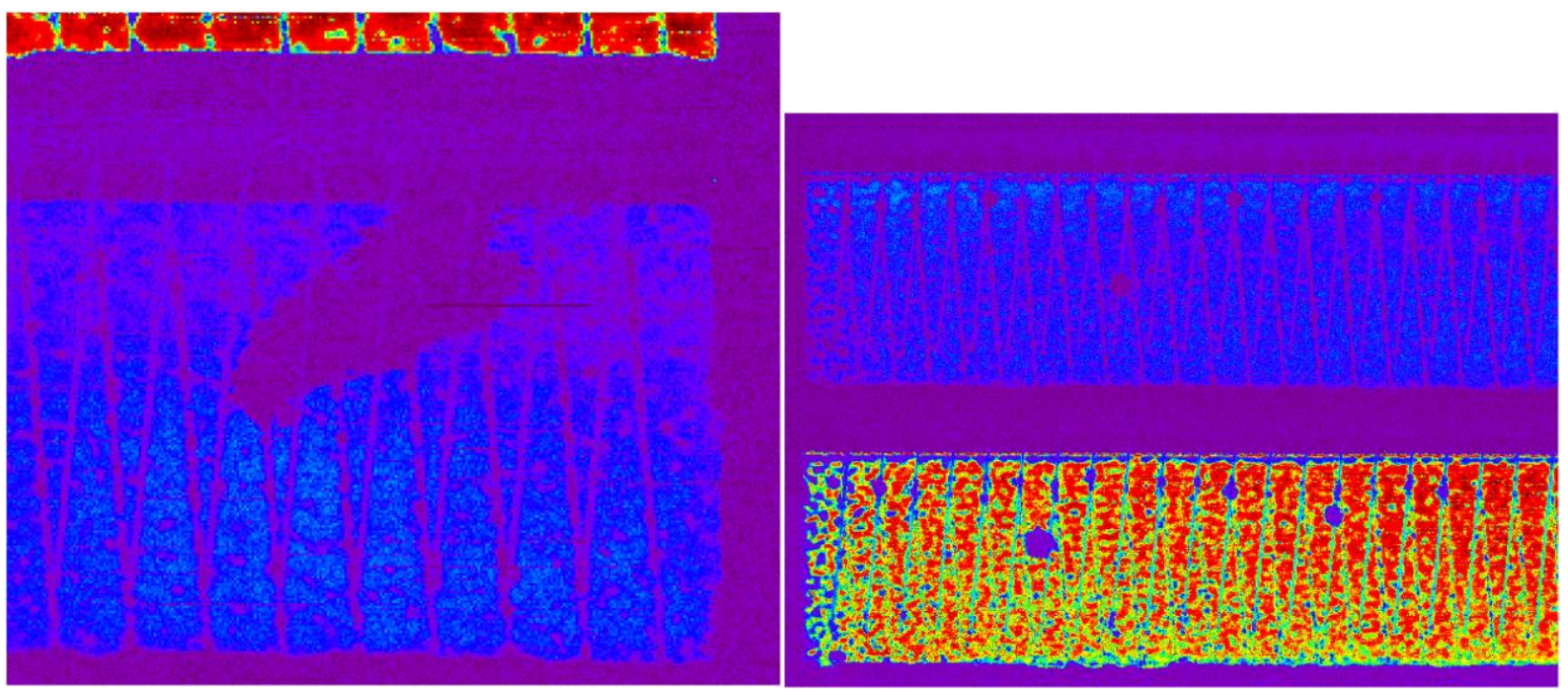

Fig. 2: $L B I C$ detail images of the defect seen on the second cell from the bottom on the right side (left image) for the ILIT image in Figure 1. And detail LBIC image of the defects found on the bottom cell left corner regarding to the ILIT image.

\section{REFERENCES}

[1] P. Apilo, M. Valimaki, R. Po, K. L. Vaisanen, H. Richter, M. Ylikunnari, M. Vilkman, A. Bernardi, G. Corso, H. Hoppe, R. Roesch, R. Meitzner, U. S. Schubert, and J. Hast, "Fully Roll-to-Roll Printed P3HT/Indene-C60Bisadduct Modules with High Open-Circuit Voltage and Efficiency", Solar RRL 2, 8 (2018). 